\title{
Magnetic processes in a collapsing dense core
}

\section{Fragmentation. Is there a fragmentation crisis?}

\author{
P. Hennebelle ${ }^{1}$ and R. Teyssier ${ }^{2}$ \\ 1 Laboratoire de radioastronomie millimétrique, UMR 8112 du CNRS, École normale supérieure et Observatoire de Paris, \\ 24 rue Lhomond, 75231 Paris Cedex 05, France \\ e-mail: patrick.hennebelle@ens.fr \\ 2 Service d'Astrophysique, CEA/DSM/DAPNIA/SAp, Centres d'Études de Saclay, 91191 Gif-sur-Yvette Cedex, France
}

Received 18 July 2007 / Accepted 17 September 2007

ABSTRACT

\begin{abstract}
Context. A large fraction of stars are found in binary systems. It is therefore important for our understanding of the star formation process, to investigate the fragmentation of dense molecular cores.

Aims. We study the influence of the magnetic field, ideally coupled to the gas, on the fragmentation in multiple systems of collapsing cores.

Methods. We present high resolution numerical simulations performed with the RAMSES MHD code starting with a uniform sphere in solid body rotation and a uniform magnetic field parallel to the rotation axis. We pay particular attention to the strength of the magnetic field and interpret the results using the analysis presented in a companion paper.

Results. The results depend much on the amplitude, $A$, of the perturbations seeded initially. For a low amplitude, $A=0.1$, we find that for values of the mass-to-flux over critical mass-to-flux ratio, $\mu$, as high as $\mu=20$, the centrifugally supported disk which fragments in the hydrodynamical case is stabilized and remains axisymmetric. Detailed investigations reveal that this is due to the rapid growth of the toroidal magnetic field induced by the differential motions within the disk. For values of $\mu$ smaller than $\simeq 5$, corresponding to higher magnetic intensities, there is no centrifugally supported disk because of magnetic braking. When the amplitude of the perturbation is equal to $A=0.5$, each initial peak develops independently and the core fragments for a large range of $\mu$. Only for values of $\mu$ close to 1 is the magnetic field able to prevent the fragmentation.

Conclusions. Since a large fraction of stars are binaries, the results of low magnetic intensities preventing the fragmentation in the case of weak perturbations is problematic. We discuss three possible mechanisms which could lead to the formation of binary systems, namely the presence of high amplitude fluctuations in the core initially, ambipolar diffusion and fragmentation during the second collapse.
\end{abstract}

Key words. magnetohydrodynamics (MHD) - instabilities - ISM: kinematics and dynamics - ISM: structure - ISM: clouds

\section{Introduction}

Understanding the fragmentation of collapsing prestellar dense core, is of great importance to our understanding of the star formation process. In particular, determining the number of fragments, their masses and their orbital characteristics are fundamental and rather challenging problems. A significant fraction of stars are found in binary or multiple systems (Duquenoy \& Mayor 1991; although see Lada 2006). Many studies have investigated this issue in the context of hydrodynamical calculations (e.g. Miyama 1992; Boss 1993; Bonnell 1994; Truelove et al. 1998; Bate \& Burkert 1997; Bodenheimer et al. 2000; Matsumoto \& Hanawa 2003; Hennebelle et al. 2004; Goodwin et al. 2004; Banerjee et al. 2004). Although detailed conclusions appear to be very sensitive to the initial conditions (i.e. core shape, thermal, rotational and turbulent energy as well as to the equations of state), some trends can nevertheless be inferred. It is widely accepted that, under realistic initial conditions, a collapsing dense core fragments into a few objects, the exact number depending on the specific conditions. Therefore, fragmentation of a rotating collapsing dense core appears to be the most widely accepted mechanism to explain the formation of multiple systems. Indeed, it is today the only viable mechanism since other possibilities such as fission of a rotating protostar or capture of a companion fail in realistic conditions to produce a large fraction of binaries (Bodenheimer et al. 2000).

Due to the difficulty of the problem and despite its importance, the question of the role of the magnetic field in this process has remained little addressed. With the recent progress achieved in numerical techniques as well as increasing computing power, several studies have investigated this issue. Hosking \& Whitworth (2003) using an SPH two fluid code conclude that magnetically subcritical cores do not fragment. Machida et al. (2005) using a nested grid code performed an extensive number of calculations varying the initial core rotation and magnetic field. They find that fragmentation is possible if the rotation is sufficiently large and the magnetic field strength sufficiently small. Fragmentation of a collapsing magnetized cloud using adaptive mesh refinement techniques have also been studied by Ziegler (2005), Banerjee \& Pudritz (2006) and Fromang et al. (2006) which all find that the magnetic field has a strong influence. Price \& Bate (2007), using magnetized SPH techniques which insure the nullity of the divergence of the magnetic field, find that with large initial perturbations, even for large values of the magnetic strength, fragmentation is possible. In all these studies, it has been found that the magnetic field has a strong impact on the fragmentation of the collapsing dense core. 
In this paper, we further study the fragmentation of collapsing cores, focusing mainly on the influence of the magnetic field strength and the amplitude of the initial perturbation. Using the analysis developed in the companion paper by Hennebelle \& Fromang (2008, hereafter Paper I), we try to understand, when possible quantitatively, the physical reasons for the numerical results. As in Paper I, we consider initially a uniform density cloud in solid body rotation, threaded by a uniform magnetic field. At this stage, we restrict the problem to the case where the magnetic field and rotation axis are aligned. We note that Price \& Bate (2007) have investigated the influence of initially perpendicular rotation axis and magnetic field. More realistic initial conditions, including a non uniform density field, turbulent velocity field and rotation axis non aligned with the magnetic field, will be considered in future studies. In particular, we determine the lowest value of $\mu$, the mass-to-flux over critical massto-flux ratio for which fragmentation is suppressed. It turns out that the results depend strongly on the amplitude of the initial perturbation.

The paper is organized as follow. In the second section, we consider the same initial conditions as used in Paper I, namely a spherical uniform one solar mass dense core. The thermal over gravitational energy of the core is equal to $\simeq 0.37$ and the rotation over gravitational energy is 0.045 . Note that such rotations are typical of (perhaps slightly larger than) values observed in dense cores (Goodman et al. 1993). The initial density is about $\simeq 5 \times 10^{-18} \mathrm{~g} \mathrm{~cm}^{-3}$ and the cloud radius $R_{0} \simeq 0.016 \mathrm{pc}$. The freefall time is thus of the order of $3 \times 10^{4}$ years. We use a barotropic equation of state: $C_{\mathrm{s}}^{2}=\left(C_{\mathrm{s}}^{0}\right)^{2} \times\left(1+\left(\rho / \rho_{\mathrm{c}}\right)^{4 / 3}\right)^{1 / 2}$, where $C_{\mathrm{s}} \simeq 0.2 \mathrm{~km} \mathrm{~s}^{-1}$ is the sound speed and $\rho_{\mathrm{c}}=10^{-13} \mathrm{~g} \mathrm{~cm}^{-3}$. This set of cloud parameters is known to give rise to a disk that unambiguously fragments in the pure hydrodynamics case. The only difference to Paper I is that an $m=2$ perturbation of amplitude $A=0.1$ in the density field, $\rho(r, \theta, z)=\rho_{0} \times(1+A \cos (m \theta))$, as well as in the $B_{z}$ field, is added. In the third section, we further discuss the physical interpretation of the results obtained in our simulations and we estimate analytically the critical value of $\mu$ for which it is expected that the magnetic field stabilizes the disk. In the fourth section, we consider initial density perturbations of amplitude 0.5 and show that in this case, fragmentation can be obtained for a much wider range of $\mu$. The fifth section provides a discussion of possible mechanisms leading to disk fragmentation and the formation of binaries, even in the presence of magnetic fields. The sixth section concludes the paper.

\section{Weak initial perturbations}

We investigate fragmentation in the case where the amplitude of the perturbations is initially $A=0.1$. In this case it is found that without rotation no fragmentation occurs. Therefore, we call this type of fragmentation, rotationally driven fragmentation. We next consider various values of the magnetic mass-to-flux over critical mass-to-flux ratio, namely $\mu=1000$ (quasi hydrodynamical case), $\mu=50,20,5,2$ and 1.25. Recalling that $\mu=1$ corresponds to the case of a cloud supported by the magnetic field, the last value corresponds to a strongly magnetized supercritical cloud.

In the following, we display the inner part of the collapsing cloud. The size of each plot is about $\simeq R_{0} / 10$. This corresponds to a physical size of $1.6 \times 10^{-3} \mathrm{pc}$ or about $\simeq 300 \mathrm{AU}$.
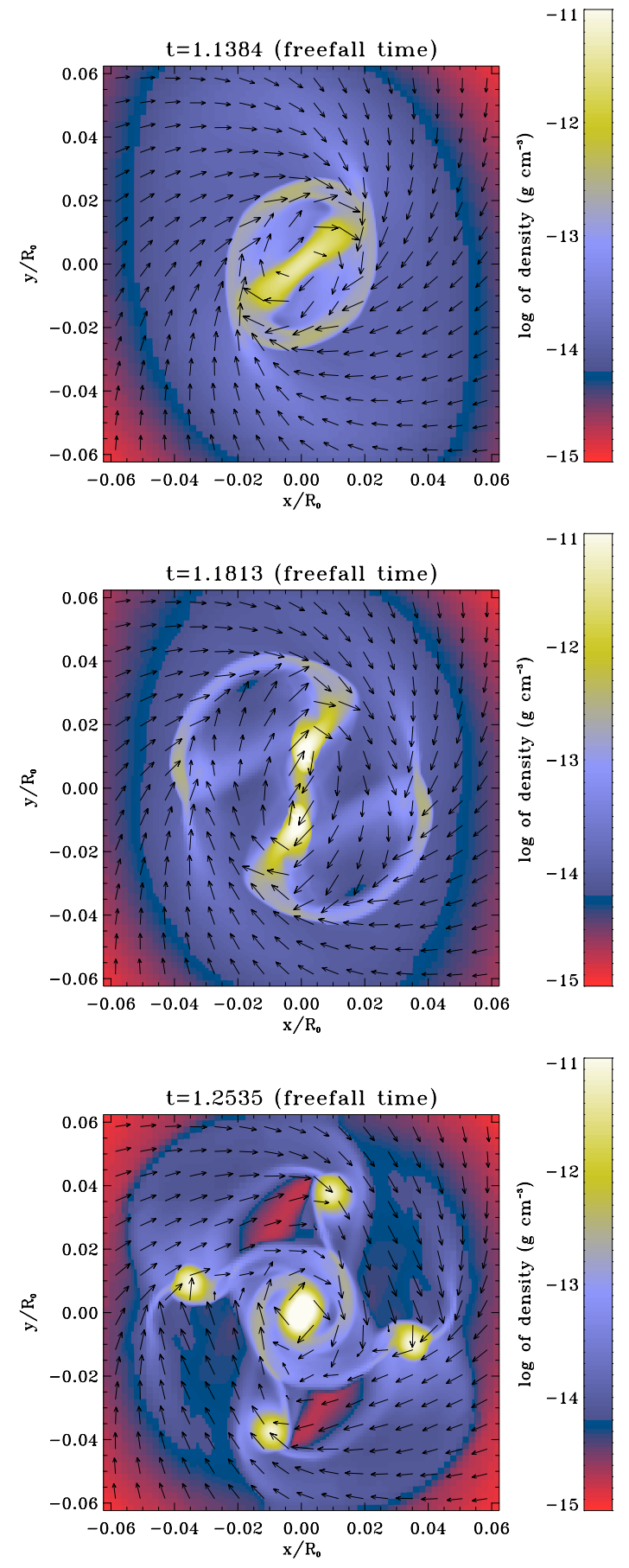

Fig. 1. Equatorial density and velocity field for mass-to-flux over critical mass-to-flux ratio $\mu=1000$ and perturbation amplitude $A=0.1$. The size of the figure corresponds to about one ten of the cloud initial radius. In physical units, this is about $300 \mathrm{AU}$. The freefall time is about $3 \times 10^{4}$ years.

\subsection{Hydrodynamic case: fragmentation}

We present the hydrodynamic case $(\mu=1000)$. Figure 1 shows three snapshots. Equatorial density and velocity field are displayed. The first snapshot is taken shortly after the formation of the first Larson core. A rotationally supported structure has formed and since it is very unstable, a strong spiral pattern develops. This is very similar to the results obtained by other authors (e.g. Matsumoto \& Hanawa 2003; Hennebelle et al. 2004; Goodwin et al. 2004). The next snapshot shows that the central 
bar-like structure fragments into two objects whereas the spiral pattern keeps growing and expanding due to further accretion of mass and momentum. The last snapshot shows that the centrifugally supported structure continues fragmenting and that 5 fragments have developed. Altogether, this is very similar to the results reported by many authors investigating the fragmentation of hydrodynamical rotating cores. We note that in spite of the strongly non-linear evolution, the result remains remarkably symmetric due to proper resolution of the Jeans length (10 numerical cells per Jeans length) despite the use of the Roe solver known to be weakly diffusive.

\subsection{Weak magnetic field cases: suppression of fragmentation}

We present results for the weak field cases, $\mu=50$ and $\mu=20$. Figure 2 shows results for $\mu=50$. Due to the difference in the initial magnetic strengths, it is not possible to compare the simulations at exactly the same times. The first snapshot shows a spiral pattern which is similar to the one seen in the previous case. A difference however, is that the central bar-like structure appears to be much shorter. This is likely due to the central magnetic field which is strongly amplified by the rapid twisting of field lines. As a result, the cloud first fragments into three fragments instead of two. This is due to the fact that since the central bar-like structure does not fragment in two objects as in the previous case, more material and angular momentum is available in its vicinity to produce one object on each side. The next snapshot shows that a symmetry breaking occurs due to one of the two satellites having merged with the central more massive fragment. The structure in the outer part $\left(x>0.03 R_{0}\right)$ is still quite symmetric. Further fragments are forming in the outer part of the spiral pattern at $x= \pm 0.05 R_{0}$.

From these results, one concludes that even for values of $\mu$ as large as 50, the magnetic field has a significant impact on the evolution of the centrifugally supported inner structure, particularly on its fragmentation. This is due to the strong amplification of the toroidal and radial magnetic field generated by the differential motions in the collapsing core and in the centrifugally supported structure (see Paper I and the following sections).

Figure 3 shows three snapshots for $\mu=20$. The first snapshot shows a weak spiral pattern in the inner part. The second and third panels shows that the centrifugally supported structure grows as in the previous cases but it remains much more uniform and the spiral pattern is much less pronounced. As shown in Paper I, the angular momentum profile is similar to the hydrodynamical case since the magnetic braking is very weak during the collapse phase. Therefore, the difference to the case $\mu=50$, is not less angular momentum but rather a stronger magnetic field. Indeed, with a magnetic toroidal component, the velocity of the fast MHD waves, which can be loosely seen as an effective sound speed, is: $\sqrt{C_{\mathrm{s}}^{2}+V_{\mathrm{a}}^{2}}$ where $V_{\mathrm{a}}^{2}=B_{\theta}^{2} / 4 \pi \rho$ (see Sect. 3). Therefore, the disk is stabilized against gravitational fragmentation. As a result, no fragmentation is obtained, only one central star forms and grows by accretion. We stress that the value of the magnetic field corresponding to $\mu=20$ is very modest, far below the values inferred from observations which indicates that $\mu=1-2$ (Crutcher 1999).

Finally, to explore whether the suppression of the fragmentation depends on initial conditions, such as the values of $\alpha$ and $\beta$ (the ratios of thermal and rotational to gravitational energies), we have performed runs with different $\alpha$ and $\beta$ values. First, we explore the influence of stronger and weaker rotation
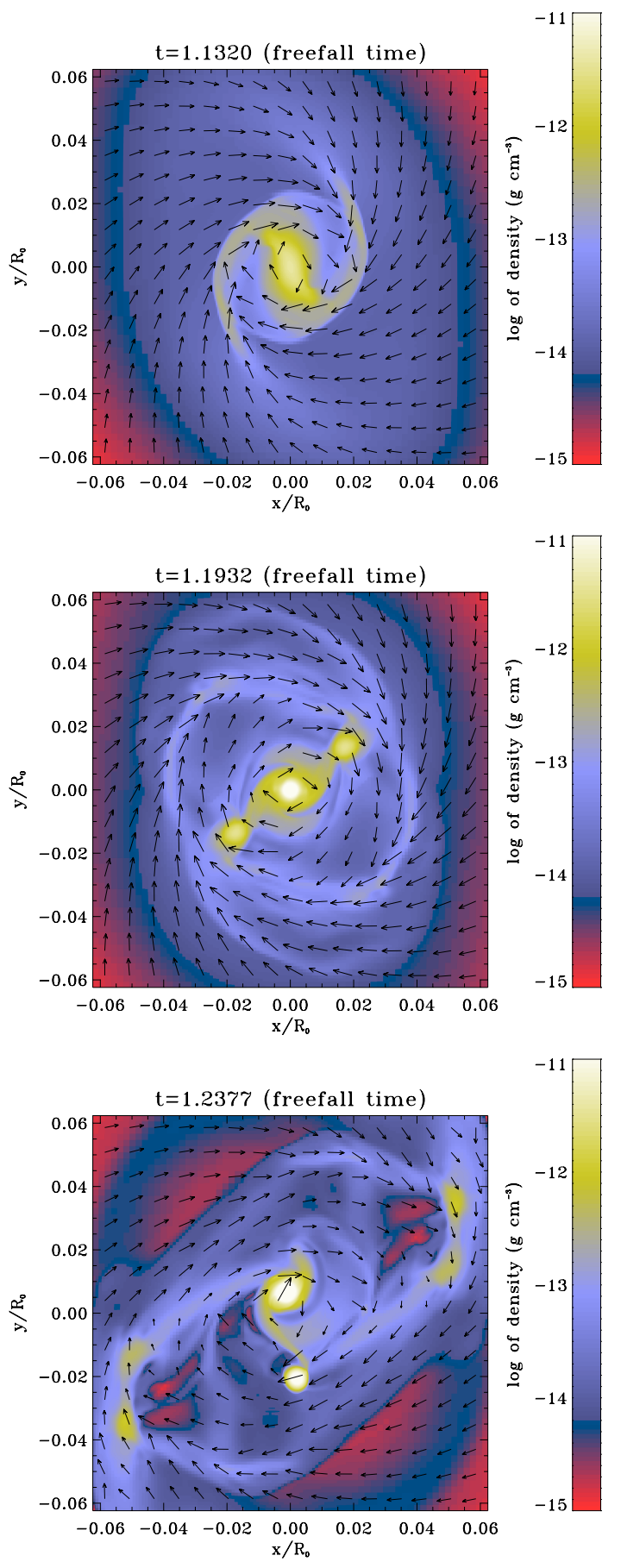

Fig. 2. Same as Fig. 1 for $\mu=50$ and $A=0.1$.

choosing $\beta=0.2$ and $\beta=0.02$ respectively, keeping the value of $\alpha$ constant. The behaviour of these runs is very similar to the one with $\beta=0.045$ presented here. A large disk forms but it remains well axisymmetric and does not fragment. We have also explored the effect of a lower thermal energy, taking $\alpha=0.2$ and keeping $\beta=0.045$. The disk is slightly more axisymmetric than in the case $\alpha=0.37$ but does not fragment either.

We conclude that, for small initial density perturbations, a magnetized dense core ideally coupled to the magnetic field and having $\mu=20$ does not fragment for a large range of initial conditions.

We compare our results with the study of Machida et al. (2005) although it is not straightforward because their initial 

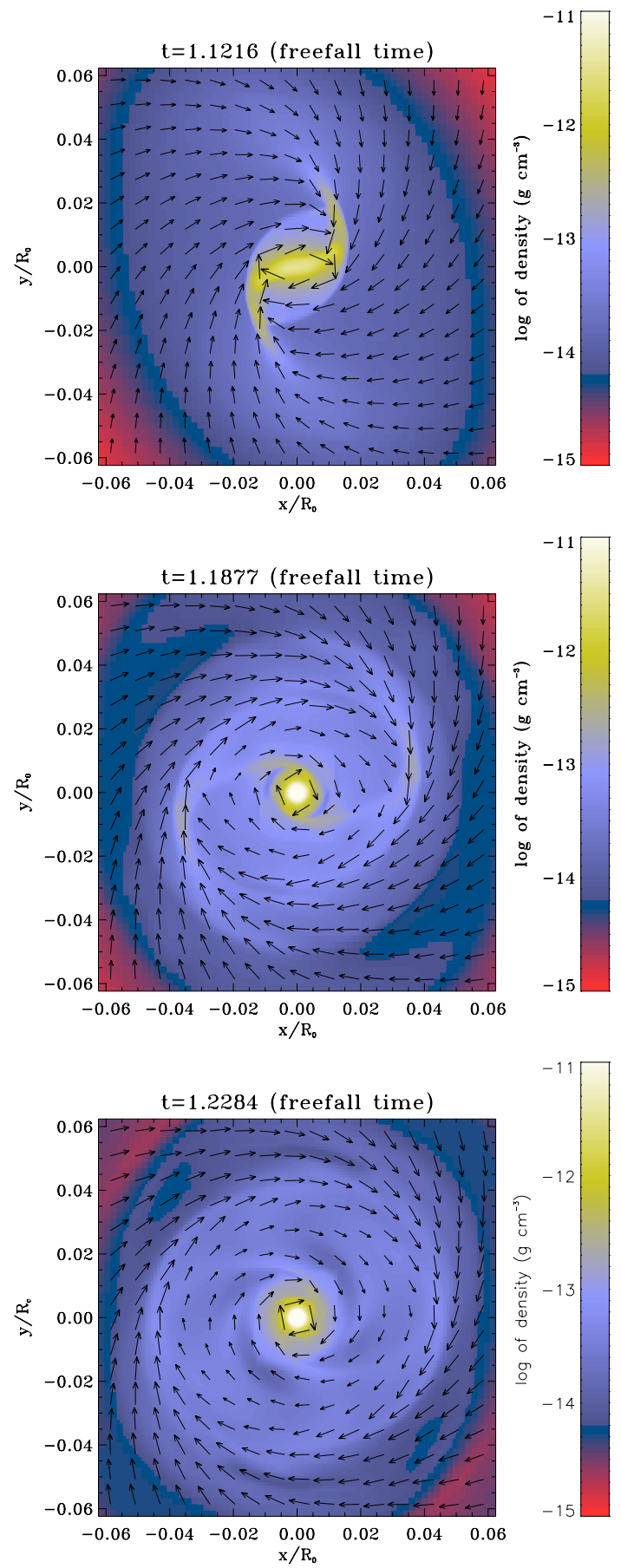

Fig. 3. Same as Fig. 1 for $\mu=20$ and $A=0.1$.

conditions consist of a filament and therefore are different from ours. Also they used different definitions to quantify the amount of rotation and magnetic energies in their simulations. Comparison with their Fig. 10 is worthwhile nevertheless. Their parameter $\omega$ turns out to be equal to $\sqrt{\beta}$. However, their initial state has a peak density of about $10^{4} \mathrm{~cm}^{-3}$ which is less dense than ours by a factor of roughly 100 . Since the ratio of rotational over gravitational energies increases during the collapse, we can say that our $\beta=0.045$ correspond to $\omega<0.2$ in Machida et al. study (for a homologous contraction $\beta \propto 1 / r \propto \rho^{-1 / 3}$, this would indicate that $\beta$ should be divided by roughly $\simeq 4-5$ to be compared with the Machida et al.'s results). For values of $\omega<0.2$, they found that for $B_{z} / \sqrt{8 \pi C_{\mathrm{s}}^{2} \rho_{\mathrm{c}}}>0.1$, they have
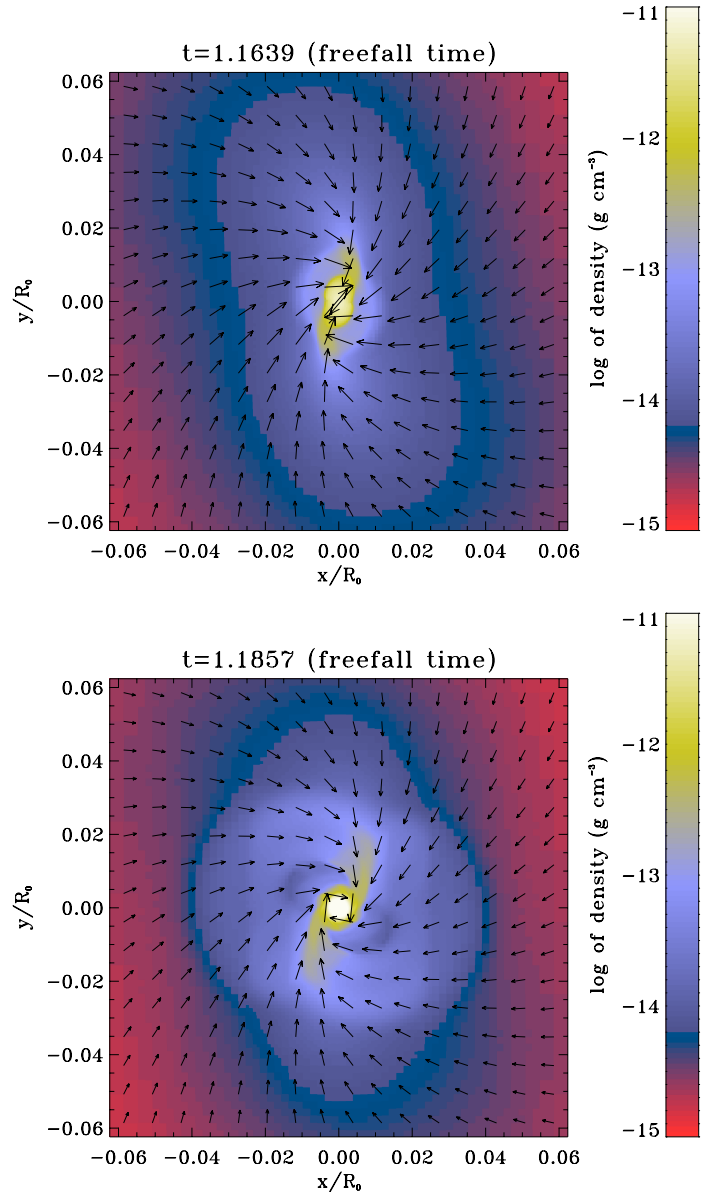

Fig. 4. Same as Fig. 1 for $\mu=5$ and $A=0.1$.

no fragmentation. Comparing this value with our parameters is again not straightforward but $B_{z} / \sqrt{8 \pi C_{\mathrm{s}}^{2} \rho_{\mathrm{c}}}$ is about $\simeq 1 / \sqrt{\alpha} \mu$. For $\alpha=0.37$ and $\mu=20,1 / \sqrt{\alpha} \mu \simeq 0.1$. Therefore, our results broadly agree with the results of Machida et al. (2005).

Comparison with the study of Price \& Bate (2007) is not possible at this stage, since they use a barotropic equation of state which becomes adiabatic at $10^{-14} \mathrm{~g} \mathrm{~cm}^{-3}$. Thus, even the hydrodynamical calculations they present do not fragment when the perturbation is weak (their Fig. 3).

\subsection{Intermediate magnetic intensity}

We present results for the intermediate magnetized cases, namely $\mu=5$ and $\mu=2$. Figure 4 shows two snapshots for $\mu=5$. As shown in Paper I, no centrifugally supported structure forms, instead a magnetized pseudo-disk develops. Pseudo-disks arise when disk-like structures form, say oblate ellipsoids, which are not supported by rotation and are produced by the magnetic field. According to the analysis presented in Paper I, the angular momentum is lower for these values of $\mu$ primarily because the collapse occurs first along the field lines. Thus, the material within the pseudo-disk and central object was initially located along the pole and has less angular momentum. Some magnetic braking also occurs, reducing the angular momentum further. As a consequence, no centrifugally supported disk is observed and no fragmentation occurs.

Figure 5 shows two snapshots for $\mu=2$. The first panel shows that a filamentary structure has developed after $t=$ 1.51 freefall times. We believe that this filament is due to the 

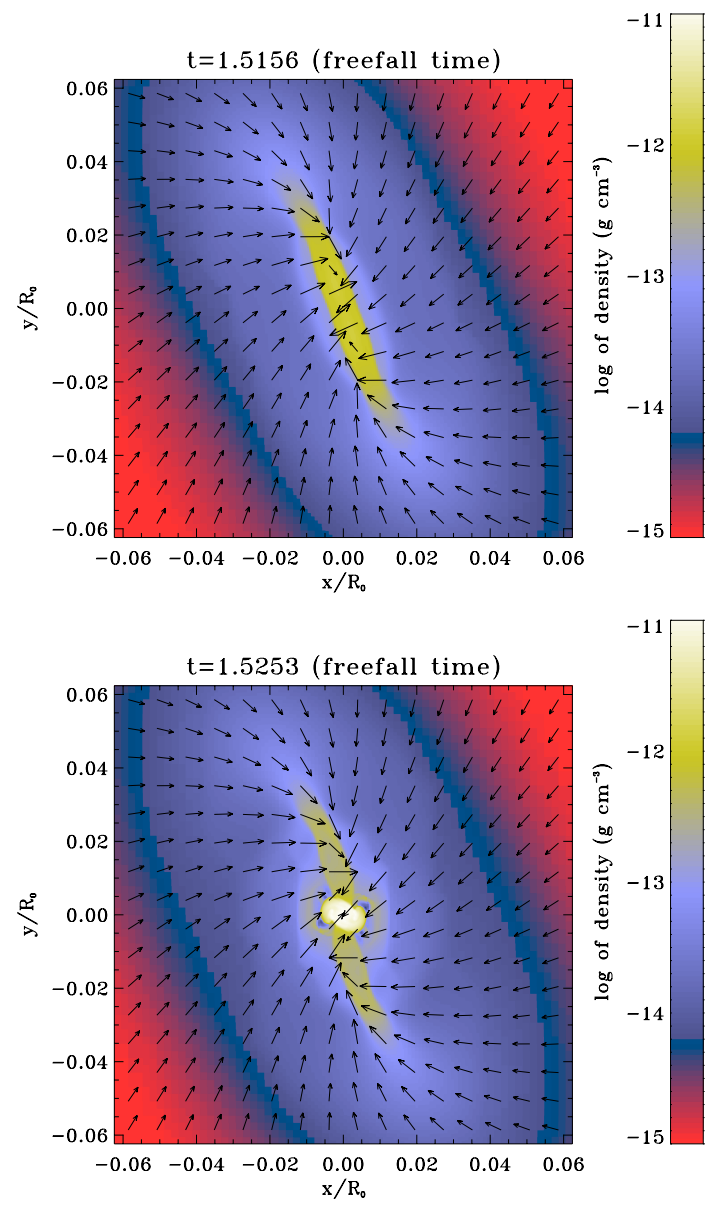

Fig. 5. Same as Fig. 1 for $\mu=2$ and $A=0.1$.

non-linear evolution of the initial $m=2$ perturbation. The reason why it has a shape different to the previous case is that since the cloud is more supported by the magnetic field, the collapse lasts $30 \%$ longer. Therefore the perturbation has more time to develop and to become non-linear. Indeed, if no perturbation is included initially, an axisymmetric pseudo-disk develops.

\subsection{Nearly critical core}

We now consider the case of a very magnetized supercritical core having $\mu=1.25$. As expected the collapsing time is now longer and roughly equal to two freefall times. As revealed by the two panels of Fig. 6, the collapsing dense core remains almost axisymmetric in spite of the initial $m=2$ density perturbations. This is due to the strong magnetic support which prevents the development of the perturbation. Indeed, since the cloud is nearly supported by the magnetic field, the magnetic Jeans Mass is just slightly smaller than the cloud mass. Therefore, each peak of the $m=2$ density perturbation is gravitationally stable. Moreover, unlike the thermal support, the magnetic support does not decrease during the collapse since the ratio of magnetic over gravitational energy stays roughly constant. Therefore the magnetic Jeans mass stays roughly constant, unlike the thermal Jeans mass.

\section{Physical interpretation}

One of the important conclusions reached in the previous section is that fragmentation can be suppressed even for large values
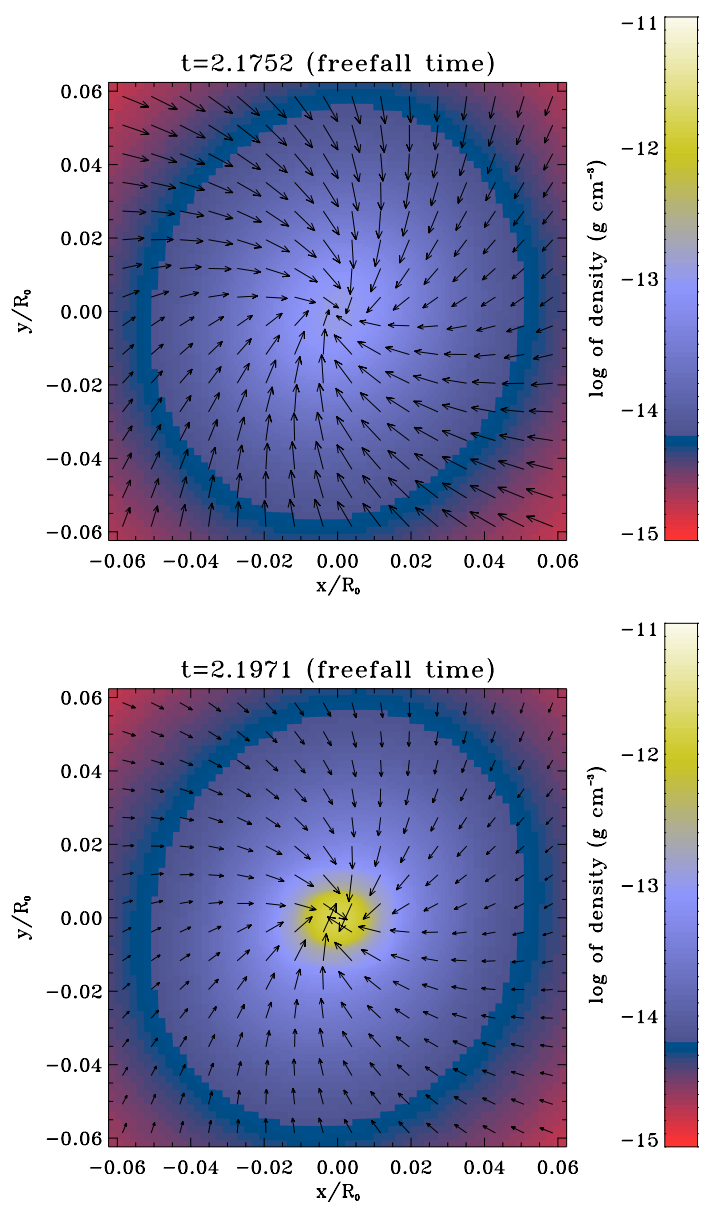

Fig. 6. Same as Fig. 1 for $\mu=1.25$ and $A=0.1$.

of $\mu$, i.e. weak magnetic fields. Here we discuss the physical reason for the disk stabilization arising at low magnetic strength. We stress that since the angular momentum is nearly identical for $\mu=20$ to the hydrodynamical case (see Paper I), the reason for this stabilization is not magnetic braking.

\subsection{Influence of the magnetic field on the disk stability}

The influence of the magnetic field on the stability of a selfgravitating disk was first investigated by Lynden-Bell (1966) for a uniform rotation. The dispersion relation he obtained (see his Eq. (1)) entails an effective sound speed $\sqrt{C_{\mathrm{s}}^{2}+V_{\mathrm{a}}^{2}}$ showing that the magnetic pressure term has a stabilizing influence. However, there is also a destabilizing contribution due to the magnetic tension term. As a result, such configurations are unstable.

Elmegreen (1987) and Gammie (1996) both consider the influence of the magnetic field on the stability of a differentially rotating system. In that case, the shear drives the growth of a toroidal magnetic component which stabilizes the disk. They both conclude that whereas in the absence of shear the magnetic field is strongly destabilizing, it has a stabilizing influence when significant shear is present. More precisely, Gammie (1996) computed the response of the disk to nonaxisymmetric perturbations for various value of $Q$, the Toomre parameter. $\mathrm{He}$ shows that the response is much weaker when a substantial magnetic field is present and concludes that the stabilizing effect of the field becomes significant once the magnetic pressure is comparable to the gas pressure. This is qualitatively in good 

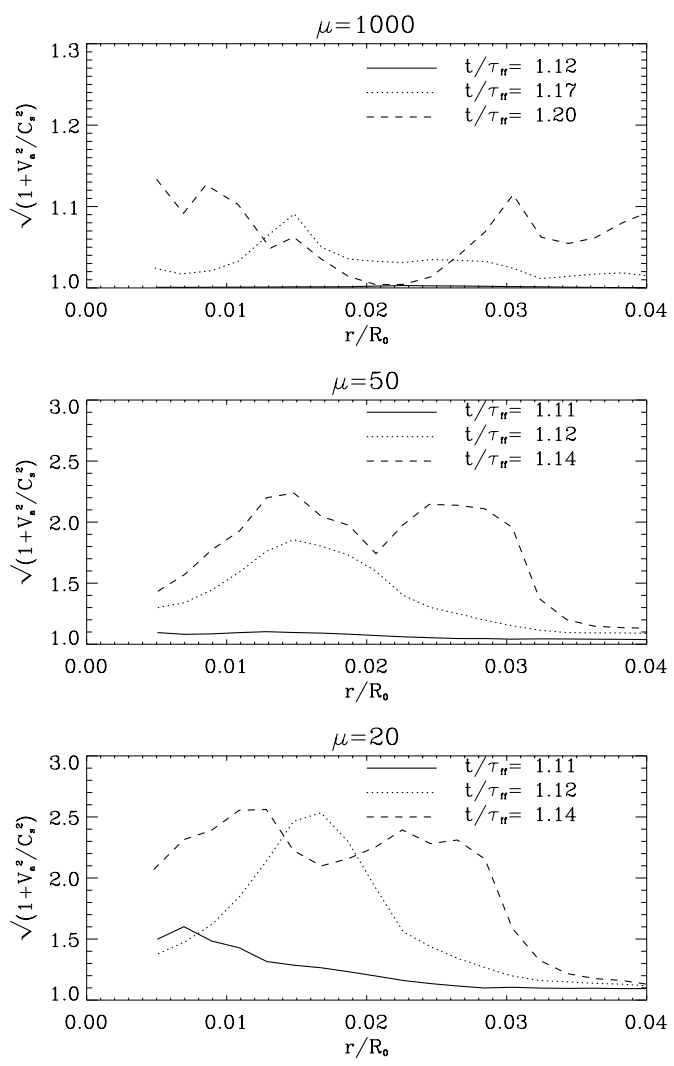

Fig. 7. $\sqrt{1+V_{\mathrm{a}}^{2} / C_{\mathrm{s}}^{2}}$ within the disk.

agreement with our results. In particular, we see that the disk in the case $\mu=20$ remains much more axisymmetric than in the case $\mu=1000$ and $\mu=50$.

In order to quantitatively verify that this mechanism is responsible for the disk stabilization, we have computed the Alfvén speed associated with the azimuthal component of the magnetic field, $B_{\theta} / \sqrt{4 \pi \rho}$, within the disk at various times corresponding to the period during which fragmentation takes place. Since both $B_{\theta}$ and $\rho$ vary, we have integrated at all radii along the $z$-axis through the disk. As explained in Paper I, as soon as the azimuthal component of the magnetic field exceeds the ram pressure of infalling material, a magnetic tower builds up and the disk starts expanding along the pole. In this case, the disk boundaries are not clearly defined. We adopt here a density threshold to define the disk and stop the integration when $\rho<10^{-14} \mathrm{~g} \mathrm{~cm}^{-3}$. We calculate $\left\langle V_{\mathrm{a}}^{2}\right\rangle=\int B_{\theta}^{2} / 4 \pi \rho \times \rho \mathrm{d} z / \int \rho \mathrm{d} z$, which allows us to estimate $\sqrt{1+\left\langle V_{\mathrm{a}}\right\rangle^{2} / C_{\mathrm{s}}^{2}}$ within the disk. Figure 7 shows $\sqrt{1+\left\langle V_{\mathrm{a}}\right\rangle^{2} / C_{\mathrm{s}}^{2}}$ for $\mu=1000,50$ and 20 . As can be seen, for the case $\mu=1000$, the quantity $\sqrt{1+\left\langle V_{\mathrm{a}}^{2}\right\rangle / C_{\mathrm{s}}^{2}}$ remains smaller than 1.2 at all the times displayed. Figure 1 shows that by this time, fragmentation has already occurred. The second and third panel of Fig. 7 show that $\sqrt{1+\left\langle V_{\mathrm{a}}^{2}\right\rangle / C_{\mathrm{s}}^{2}}$ grows much more rapidly for smaller $\mu$ reaching values greater than 2 . The growth is more rapid for $\mu=20$ than it is for $\mu=50$. We conclude that the growth of the toroidal component induced by the differential rotation is mainly responsible for disk stabilization.

For simplicity, the works mentioned above have restricted the analysis to a thin disk. However, the expansion of the magnetic tower triggered by the growth of the toroidal magnetic field removes some material from the disk and therefore reduces the disk surface density. This makes the disk even less prone to fragmentation. This effect, which is not taken into account in the thin disk analysis, certainly contributes to further stabilize the centrifugally supported structure against fragmentation. Indeed, the mass of the disk and the mass within the tower, are roughly comparable.

\subsection{Analytical estimate of the critical value of $\mu$}

We estimate analytically the value of $\mu$ at which one expects to find a significant influence of the magnetic field on the fragmentation. Following Gammie (1996), we will consider that a strong stabilizing influence is achieved when the Alfvén speed associated with the toroidal component is comparable to the sound speed.

The principle of the analysis is as follow: we compute the growth rate of the toroidal component inside the centrifugally supported structure, so that we can estimate the time, $\tau_{\text {mag }}$, needed for the Alfvén speed to become comparable to the sound speed. We then compute the dynamical time of the disk, $\tau_{\text {dyn }}$, over which fragmentation will occur. The critical value of $\mu$, below which fragmentation is quenched, is obtained when $\tau_{\text {dyn }}$ is equal to $\tau_{\text {mag }}$.

The growth time of the toroidal magnetic component within the disk has two contributions. First the twisting of the radial component due to differential rotation proportional to $V_{\theta} B_{r} / r$ and second the wrapping of the vertical magnetic component due to the vertical gradient of $B_{z}$ proportional to $V_{\theta} B_{z} / z$, where $z$ is the disk height. The divergence constraint shows that $B_{r} / r \simeq$ $B_{z} / z$, indicating that the 2 contributions are comparable.

Thus, the growth of $B_{\theta}$ can be estimated by writing $\partial_{t} B_{\theta} \simeq$ $B_{z} V_{\theta} / h$ where $h$ is the disk height. Since we are interested in computing the growth of the magnetic field from initially small values, we neglect the influence of the magnetic tower on the disk vertical expansion and write: $h \simeq \sqrt{C_{\mathrm{S}}^{2} /(4 \pi G \rho)}$. With Eq. (1) of Paper I, we obtain $h \simeq r / \sqrt{2 d}$, where $d$ is the ratio of the density over singular isothermal density.

If we assume that the disk is roughly Keplerian, we get $V_{\theta} \simeq$ $\sqrt{M_{\mathrm{s}} G / r}, M_{\mathrm{s}}$ being the mass of the star and the disk. Taking the expression of $B_{z}$ stated by Eqs. (1) of Paper I, we obtain:

$B_{\theta} \simeq t \times \frac{\sqrt{2 d} H_{z} \sqrt{M_{\mathrm{s}}} C_{\mathrm{s}}^{2}}{r^{5 / 2}}$

The value of $\tau_{\mathrm{mag}}$, is obtained by requiring $\left(B_{\theta} / \sqrt{4 \pi \rho}\right) / C_{\mathrm{s}} \simeq 1$. Using the expression of density stated by Eq. (1) of Paper I, we obtain:

$\tau_{\mathrm{mag}} \simeq \frac{r^{3 / 2}}{H_{z} \sqrt{G M_{\mathrm{s}}}}$

The dynamical time of the disk, i.e. the time relevant for fragmentation, is the rotation time:

$\tau_{\text {dyn }} \simeq \frac{2 \pi r^{3 / 2}}{\sqrt{G M_{\mathrm{s}}}}$.

Therefore, we obtain:

$\frac{\tau_{\mathrm{mag}}}{\tau_{\mathrm{dyn}}} \simeq \frac{1}{2 \pi H_{z}}$

To compute the value of $H_{z}$, one can use Table 1 of Paper I, but it is more convenient to express the result in terms of the initial cloud parameters, namely $\mu$ and $\alpha$. The latter is the ratio of thermal over gravitational energy and is given by $\alpha=$ $5 / 2 C_{\mathrm{s}}^{2} R_{0} /(M G)$. From Eq. (4) of Paper I, the value of $H_{z}$ is given 
by $\sqrt{G} B_{z}^{0} R_{0} /\left(2 C_{\mathrm{s}}^{2}\right)$, whereas from the definition of $\mu, B_{z}^{0}$ can be written as $M_{0} /\left(\pi R_{0}^{2}\right) \sqrt{G} \sqrt{9 \pi^{2} / 5} /(0.53 \mu)^{1}$. Gathering the different expressions, we get:

$\frac{\tau_{\mathrm{mag}}}{\tau_{\mathrm{dyn}}} \simeq \frac{\sqrt{2}}{3 \sqrt{5} \pi} \times \mu \alpha$.

We can deduce the critical value of $\mu$

$\mu_{\mathrm{c}} \simeq \frac{15}{\alpha}$.

Note that this value has not been derived using a rigorous analysis. It is therefore approximate and should be valid within a factor of a few.

For $\alpha \simeq 0.37$, we obtain $\mu_{\mathrm{c}} \simeq 40$. This analytical estimate is therefore in reasonable agreement with our numerical simulation, since for $\mu=20$, fragmentation is suppressed. In this case, the toroidal magnetic pressure becomes comparable to the sound speed in about half a rotation period. Since typically fragmentation occurs over a few rotation period, such a fast growth time appears to be sufficient to prevent the disk from fragmenting. On the other hand for $\mu=50$ the disk fragments, confirming that this case is slightly above the critical value.

For $\mu=1000$, one has $\tau_{\mathrm{mag}} / \tau_{\mathrm{dyn}} \simeq 20$. Therefore in such a case, the growth of the toroidal component is too slow to significantly influence the disk evolution.

\section{Strong initial perturbations}

In this section, we investigate the effect of a density fluctuation having a larger initial amplitude, $A=0.5$. In that case the cloud is more prone to fragmentation because the perturbations develop quickly. Such strong perturbations can be due either to initial conditions or to strong external triggering. Although in this section we keep the same amount of rotation as in the previous section, we also performed simulations with the same initial conditions but with no rotation and found similar results during the early phase of fragmentation to the case with rotation. This indicates that with such strong perturbations, the initial cloud fragmentation is purely thermal and independent of the rotation. We refer to this situation as thermal fragmentation.

\subsection{Thermal fragmentation}

We present results for weak and intermediate values of magnetic strengths, namely $\mu=20$ and $\mu=2$. Figure 8 shows results for $\mu=20$. The first panel shows that a filamentary structure develops in which two protostars have formed. The two protostars are well separated by roughly $\simeq 0.15 \times R_{0}$ as opposed to $\simeq 0.01 \times R_{0}$ in the case of weaker perturbations. As explained previously, the fragmentation in this case has a purely thermal origin and is simply due to the development of the initial perturbations (in the case of weak perturbations, rotationally driven fragmentation does not strongly depend on the perturbation amplitude). The second panel shows that the two fragments are approaching each other falling along the filament. The final panel shows that the two fragments have merged and that a protostellar disk

1 The value of $H_{z}$ used here is the value of the envelope whereas strictly speaking the value of $H_{z}$ that one should use is the value within the disk. Since in practice, it is typically only few times larger (Fig. 2 of Paper I) and since we do not have analytical expression for the value in the disk, we use the value in the envelope.
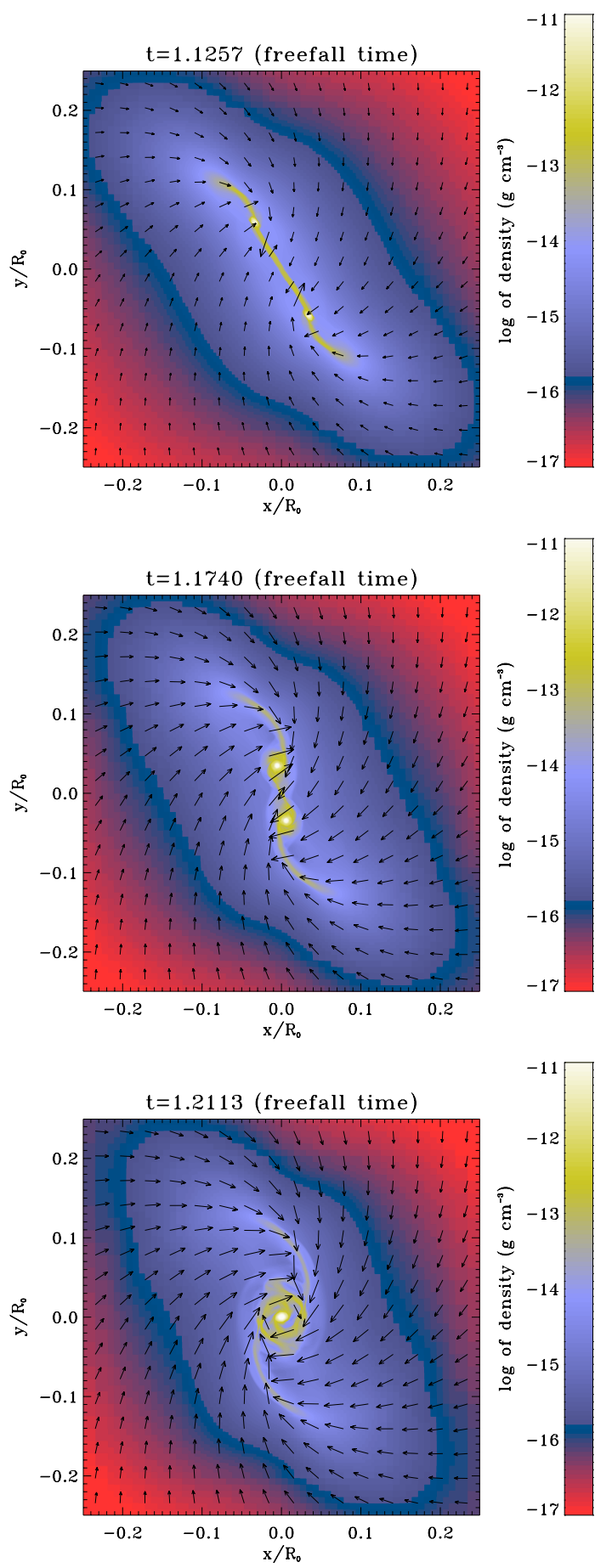

Fig. 8. Same as Fig. 1 for $\mu=20$ and $A=0.5$. The figure corresponds to a length of $\simeq 1200 \mathrm{AU}$, four times bigger than the length displayed in Fig. 1.

forms. However, the second collapse is not treated in the simulation, thus only the first Larson core is considered. Therefore the merging should be considered with great care. Careful thermodynamic treatment of the first Larson core is required. Indeed the first core is known to have a short life time of roughly $10^{3}$ years. Therefore since the timeshift between the first and second panel is larger than $10^{4}$ years, it is expected that second collapse would have already occurred by the time the two fragments approach each other. Since stars have very small radii, it is unlikely that the binary will merge. Therefore the final product could be a binary rather than a single protostar. 

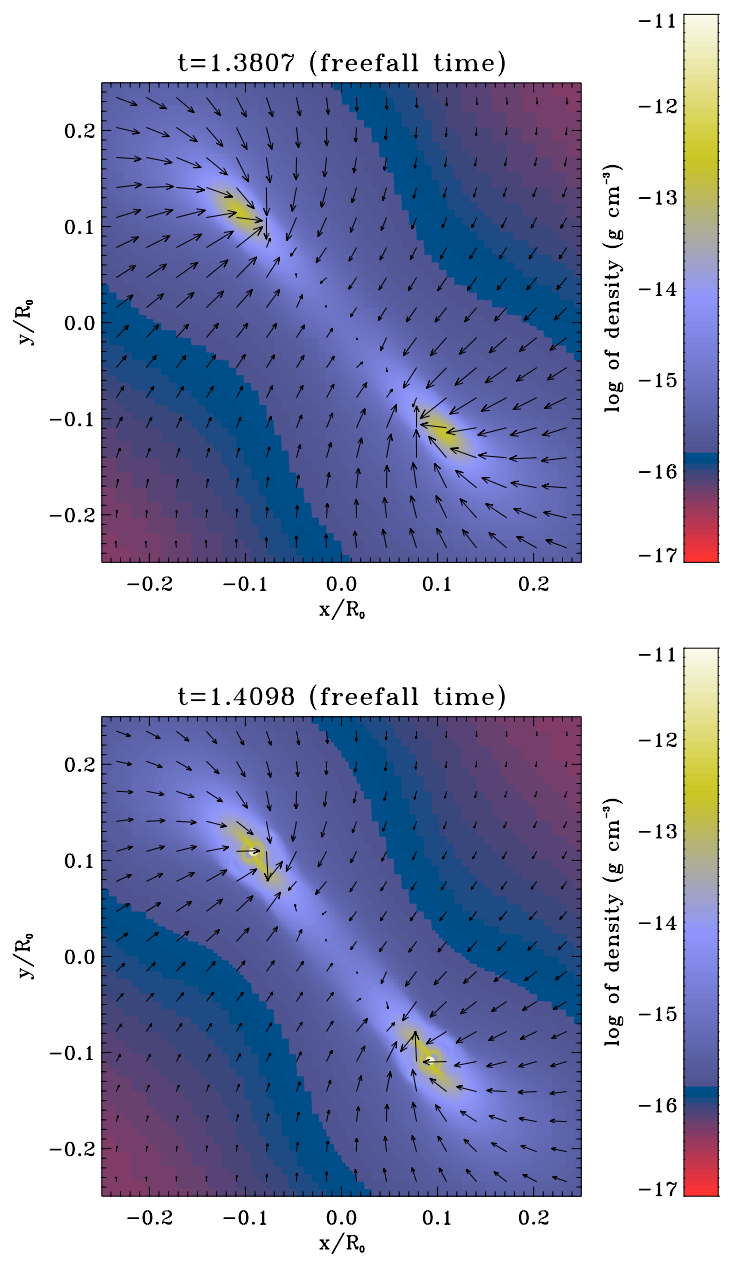

Fig. 9. Same as Fig. 1 for $\mu=2$ and $A=0.5$.

Figure 9 shows results for $\mu=2$. Both panels show that two fragments develop in a similar way as for $\mu=20$. Unfortunately, the relative strong value of the magnetic intensity makes the time steps much shorter than in the previous case and it becomes computationally expensive to follow the long range evolution. However, it seems likely that a similar evolution is expected leading to the merging and the two first cores.

\subsection{Strong field: Suppression of thermal fragmentation}

Figure 10 shows results for $\mu=1.25$ and $A=0.5$. As revealed by the first panel, the $m=2$ density perturbation has only weakly developed and only two shallow density maxima are visible. The second panel shows a single object in the centre surrounded by a pseudo-disk. Since the timeshift between the two panels is about 0.015 freefall time $\left(\simeq 5 \times 10^{2}\right.$ years $)$ there is not sufficient time for the second collapse to arise. It seems plausible that in this particular case, a single star will form.

Therefore, it appears that a nearly critically magnetized dense core, ideally coupled to the magnetic field, is difficult to fragment into a few objects even if relatively large fluctuations are initially present. This conclusion is in good agreement with the conclusion reached by Hosking \& Whitworth (2004), that a magnetically subcritical cloud, which collapses after sufficient magnetic flux has been lost because of ambipolar diffusion, does not fragment.

We conclude that, with large amplitude density fluctuations, a collapsing dense core fragments over a large range of initial
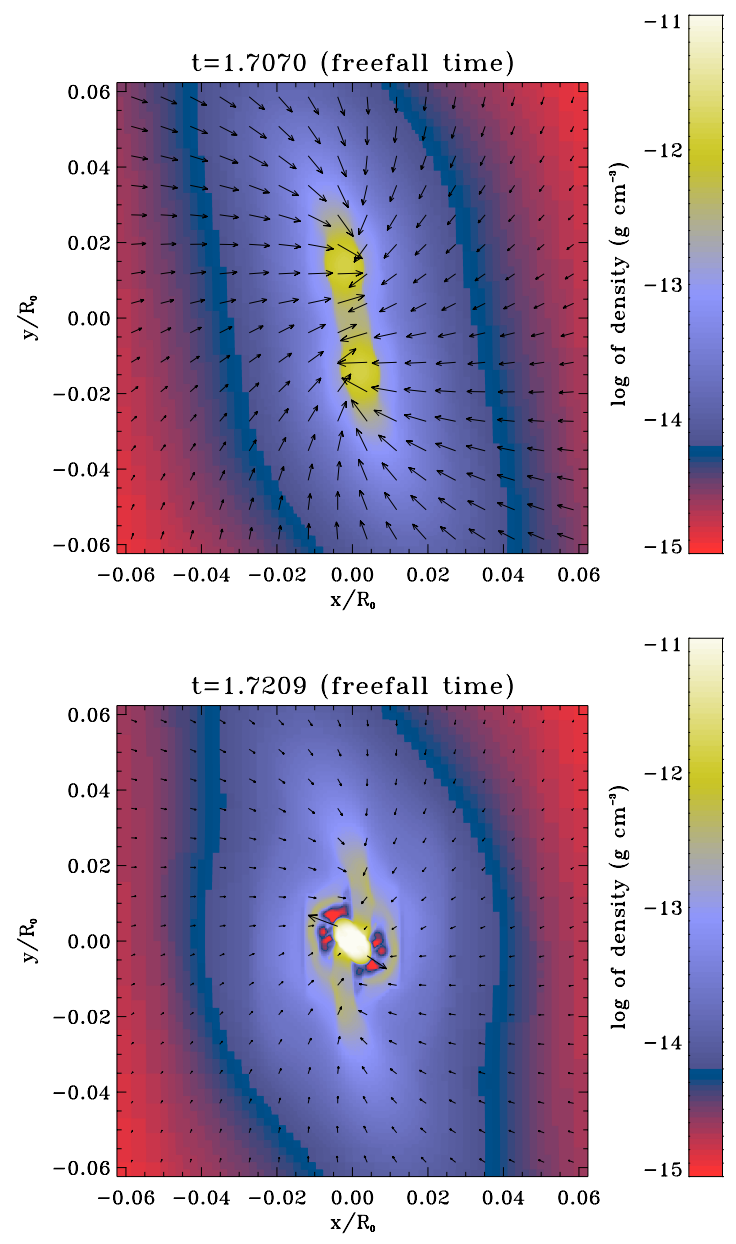

Fig. 10. Same as Fig. 1 for $\mu=1.25$ and $A=0.5$.

conditions as long as it is not too strongly magnetized. The fragments are due to each initial seed collapsing individually. The question of the survival of the fragments should however be carefully investigated. We note that Price \& Bate (2007) reach very similar conclusions. They also propose that in some cases, such as when the magnetic field is perpendicular to the initial rotation axis, the magnetic field may help the fragments to survive.

\section{Discussion}

Since there is no question that a significant fraction of stars are binaries, the efficient stabilization of the disk and the suppression of fragmentation even for modest field amplitudes may constitute a severe difficulty. We have no answer to this apparent conundrum but we discuss three possible mechanisms that may induce the fragmentation of a dense core and the formation of binaries. We also suggest various observational tests that could discriminate between these scenarios.

\subsection{Large initial density fluctuations}

We start with the possibility of having initial sufficiently large fluctuations. As demonstrated in this paper and in Price \& Bate (2007), fragmentation is possible in such a situation. The question is then how likely are large initial perturbations? Observationally the question is not easy to address. It is nevertheless known that the dense cores usually are not very 
uniform and that typically sonic velocity dispersion is observed. This is broadly compatible with the presence of density perturbations although perhaps not as large as $50 \%$. On the other hand, cores with a high aspect ratio are observed. If some of them are elongated, i.e. prolate objects, this may be equivalent to a substantial $m=2$ perturbation. Indeed, simulations of quiescent cores having transonic turbulence present significant fluctuations (Goodwin et al. 2004). It seems however difficult at this stage to infer quantitatively whether the perturbations generated by this weak turbulence are sufficient to produce multiple systems.

Another possibility is that the collapse may be induced by an external agent such as a supernova remnant or protostellar jet. These are likely to generate large perturbations. Indeed, all simulations considering clouds evolving far from equilibrium (Bate et al. 2003; Ballesteros-Paredes et al. 2003; Hennebelle et al. 2006; Peretto et al. 2007) do find dense cores with initially strong perturbations.

Future works should specify under what conditions such initial fluctuations are likely to be produced. This may depend on the physical properties of the molecular clouds in which the dense core is embedded. For example, the answer could be different in the Taurus molecular cloud in which star formation is relatively quiescent and in the Orion molecular cloud where star formation is more active.

We stress that this mechanism constitutes a change of paradigm with respect to the standard hydrodynamical scenario (corresponding to Fig. 1). Indeed, with this mechanism the fragmentation appears to be the result of perturbations seeded at large scales rather than an intrinsic properties of the collapsing dense core.

An important prediction of this model is that the binary should not necessarily be located in the equatorial plane of the core since the initial perturbations likely should be randomly distributed.

\subsection{Ambipolar diffusion}

In the present calculations, perfect coupling between gas and magnetic field is assumed. The loss of flux due to ambipolar diffusion could therefore help to reduce the magnetic intensity. The ratio of the ambipolar diffusion time, $\tau_{\text {ad }}$, and the freefall time, $\tau_{\text {ff }}$, has been computed by Shu et al. (1987). When the magnetic field is just sufficient to compensate for gravity, they obtain $\tau_{\mathrm{ad}} / \tau_{\mathrm{ff}} \simeq 8$, indicating that the ambipolar diffusion time is larger than the freefall time. When the magnetic field is weaker, the ambipolar time increases further. Therefore, it appears unlikely that the magnetic field could be removed from the envelope of a supercritical collapsing core.

However, the possibility remains that ambipolar diffusion could occur in the disk allowing the toroidal magnetic field to be diffused out. To investigate this issue, we estimate the ratio of ambipolar diffusion time in the disk along the vertical direction and the growth time of the toroidal magnetic field given by Eq. (2). Here we write it as

$\tau_{\mathrm{mag}} \simeq \frac{\sqrt{4 \pi \rho} h C_{\mathrm{s}}}{B_{z} V_{\theta}}$.

The ambipolar diffusion time of the toroidal component along the vertical direction is:

$\tau_{\mathrm{ad}} \simeq \frac{4 \pi \gamma \rho \rho_{\mathrm{i}} h^{2}}{B_{\theta}^{2}}$, where $\gamma=3.5 \times 10^{13} \mathrm{~cm}^{3} \mathrm{~g}^{-1} \mathrm{~s}^{-1}$ is the drag coefficient and $\rho_{\mathrm{i}}$ the density of ions. Since we are interested in the value of $B_{\theta}$ such that $B_{\theta} / \sqrt{4 \pi \rho} \simeq C_{\mathrm{s}}$, the ambipolar diffusion time becomes:

$\tau_{\mathrm{ad}} \simeq \frac{\gamma \rho_{\mathrm{i}} h^{2}}{C_{\mathrm{s}}^{2}}$

following Shu et al. (1987), we write $\rho_{\mathrm{i}}=C \sqrt{\rho}$ where $C=$ $3 \times 10^{-16} \mathrm{~cm}^{-3 / 2} \mathrm{~g}^{1 / 2}$. With $h \simeq r / \sqrt{2 d}, V_{\theta}=\sqrt{M_{\mathrm{s}} G / r}$ and $B_{z}=H_{z} C_{\mathrm{s}}^{2} / \sqrt{G} r$, we obtain

$\frac{\tau_{\mathrm{ad}}}{\tau_{\mathrm{mag}}}=\frac{\gamma C}{(4 \pi)^{1 / 2} C_{\mathrm{s}}} \sqrt{\frac{M_{\mathrm{s}}}{r}} \frac{H_{z}}{\sqrt{2 d}}$.

Thus, we obtain

$\frac{\tau_{\mathrm{ad}}}{\tau_{\mathrm{mag}}}=36 \times \sqrt{\frac{M_{\mathrm{s}}}{0.1 M_{\odot}}} \sqrt{\frac{100 \mathrm{AU}}{r}} \frac{H_{z}}{\sqrt{d}}$.

For $\mu=20, H_{z} \simeq 0.4$. From Fig. 2 of Paper I, $d \simeq 10$. Thus, the ambipolar diffusion time is longer than the growth time of the toroidal component. It is therefore likely that ambipolar diffusion will not change the picture significantly except if stiffer field variations than the one calculated above are present. In this case, ambipolar diffusion could help clouds with low $\mu$ to fragment more easily. We do not expect it however, to shift very significantly the value of $\mu$ for which fragmentation occurs. Since $H_{z}$ is proportional to $1 / \mu, \tau_{\mathrm{ad}} / \tau_{\mathrm{mag}}$ increases rapidly when $\mu$ decreases.

\subsection{Fragmentation during the second collapse}

Fragmentation during the second collapse has been investigated by Bonnell \& Bate (1994) with the prospect of exploring whether the formation of close binary systems could be possible. They conclude that fragmentation is indeed possible but that the binaries have to accrete most of their mass since they are initially very small.

On the other hand, Machida et al. (2007) recently explored magnetized second collapse taking into account the large Ohmic dissipation (we note that this is not rigorously Ohmic dissipation) that is predicted to occur in the first Larson core by various models (e.g. Nakano et al. 2002). As a result, most of the magnetic flux is lost making fragmentation easier. It therefore appears possible that fragmentation could occur during this phase, possibly driven by rotation. In this case, the binary should gain sufficient angular momentum from the accretion to increase the separation between the two stars (see e.g. Goodwin et al. 2004). Interestingly, Banerjee \& Pudritz (2006) report the formation of a very close binary in their second collapse calculations despite the ideal MHD assumption.

The prediction of this model is that the binaries should be in the equatorial plane since the orbital angular momentum of the binary is due to the angular momentum of the accreting gas. Also the separation between the 2 stars should increase with time implying that closer binaries should be observed on average in younger cores. Note that this mechanism could possibly work even if the magnetic field is initially very strong in the core.

In order to test each of these scenarios, high resolution observations would be necessary. In this respect, ALMA will certainly be a very powerful tool, as demonstrated by the synthetic observations performed by André et al. (2007) using the present calculations. 


\section{Conclusion}

We have studied the fragmentation of a collapsing magnetized molecular dense core by performing a set of numerical simulations. We interpret the results together with the analysis performed in the companion paper in which we investigate the accretion and ejection processes that take place in the simulations.

With our choice of initial conditions, and for perturbations of low amplitude, unmagnetized cores do fragment, while magnetized cores having values of $\mu$ as large as 20 do not fragment. Based on an analytic estimate, we suggest that for cores having $\mu$ larger than $\simeq 15 / \alpha$, the growth rate of the toroidal component is too slow to stabilize the disk. We stress that the suppression of fragmentation in this range of parameter, is not due to magnetic braking but to the rapid growth of the toroidal component of the magnetic field induced by the differential rotation within the disk. The Alfvén speed associated with this toroidal component adds up to the sound speed of the disk and stabilize it.

For values of $\mu$ smaller than 5, no big centrifugal disk forms because first the collapse occurs mainly along the field lines bringing less angular momentum, and second, magnetic braking removes angular momentum. This makes these cores even less prone to fragment.

The situation is different if large amplitude perturbations are initially seeded. In this case, each perturbation develops independently even without rotation. Since the strong field amplification is primarily due to differential motions in the disk, the magnetic field is unable to suppress fragmentation except if the core is almost critical, i.e. the field is initially very strong. The following evolution of these fragments requires a careful treatment of the thermodynamics of the first Larson core. It is indeed likely that if the second collapse has not occurred by the time these fragments approach each other, they will merge. On the other hand, if the protostars have already formed, the binary system is likely to survive.

For dense cores having rotation and magnetic strength typical of values inferred from observations, we find that fragmentation is suppressed by the magnetic field if the initial density perturbations are too small. This constitutes a severe problem, since there is no question that a significant fraction of stars are binaries. In view of this, we discuss the likelihood of having sufficient perturbations within the cores initially as well as the impact of ambipolar diffusion and the possibility of fragmentation during the second collapse phase. We speculate that the first may depend on the physical characteristic of the molecular cloud in which the dense core is embedded whereas the latter should be relatively independent of the large scales and could work even if the magnetic field is initially very strong.

Acknowledgements. Some of the simulations presented in this paper were performed at the IDRIS supercomputing center and on the CEMAG computing facility supported by the French Ministry of Research and Education through a Chaire d'excellence awarded to Steven Balbus. We thank Sébastien Fromang, Doug Johnstone and Philippe André for a critical reading of the manuscript. We thank Frank Shu, the referee, for helpful comments. P.H. thanks Masahiro Machida for related discussions.

\section{References}

André, P., Hennebelle, P., \& Peretto, N. 2007, Ap\&SS, ed. R. Bachiller et al. (special issue ALMA), in press

Ballesteros-Paredes, J., Klessen, R., \& Vazquez-Semadeni, E. 2003, Ap, 592, 188

Banerjee, R., \& Pudritz, R. 2006, Ap, 641, 949

Banerjee, R., Pudritz, R., \& Holmes, L. 2004, MNRAS, 355, 248

Bate, M., \& Burkert, A. 1997, MNRAS, 288, 1060

Bate, M., Bonnell, I., \& Bromm, V. 2003, MNRAS, 339, 577

Bodenheimer, P., Burkert, A., Klein, R., \& Boss, A. 2000, in Protostars and Planets IV, ed. V. Mannings, A. P. Boss, \& S. S. Russell (Tucson: Univ. of Arizona Press), 675

Bonnell, I. 1994, MNRAS, 269, 837

Bonnell, I., \& Bate, M. 1994, MNRAS, 271, 999

Boss, A. 1993, Ap, 410, 157

Crutcher, R. 1999, Ap, 520, 706

Duquenoy, A., \& Mayor, M. 1991, A\&A 248, 485

Elmegreen, B. 1987, Ap, 312, 626

Fromang, S., Hennebelle, P., \& Teyssier, R. 2006, A\&A, 457, 371

Gammie, C. 1996, Ap, 462, 725

Goodman, A., Benson, P., Fuller, G., \& Myers, P. 1993, Ap, 406, 528

Goodwin, S., Whitworth, A., \& Ward-Thompson, D. 2004, A\&A, 423, 169

Hennebelle, P., \& Fromang, S. 2008, A\&A, 477, 9 (Paper I)

Hennebelle, P., Whitworth, A., Aha, S.-H., \& Goodwin, S. 2004, MNRAS, 340, 870

Hennebelle, P., Whitworth, A., \& Goodwin, S. 2006, A\&A, 451, 141

Hosking, G., \& Whitworth, A. 2004, MNRAS, 347, 994

Lada, C. 2006, ApJ, 640, L63

Lynden-Bell, D. 1966, Observatory, 86, 57

Machida, M., Matsumoto, T., Hanawa, T., \& Tomisaka, K. 2005, MNRAS, 362, 382

Machida, M., Inutsuka, S.-i., \& Matsumoto, T. 2007, ApJ, submitted [arXiv: astro-ph/07052073]

Matsumoto, T., \& Hanawa, T. 2003, ApJ, 595, 913

Miyama, S. 1992, PASJ, 44, 193

Nakano, T., Nishi, R., \& Umebayashi, T. 2002, ApJ, 573, 199

Peretto, N., Hennebelle, P., \& André, P. 2007, A\&A, 464, 983

Price, D., \& Bate, M. 2007, MNRAS, 377, 77

Shu, F., Adams, F., \& Lizano, S. 1987, ARA\&A, 25, 23

Teyssier, R. 2002, A\&A, 385, 337

Truelove, J., Klein, R., McKee, C., et al. 1998, ApJ, 495, 821

Ziegler, U. 2005, A\&A, 435, 385 